\title{
Mechanical Properties of Human Coronary Arteries
}

\author{
E. Claes, J.M. Atienza, G.V. Guinea, F.J. Rojo, J.M. Bernal, J.M. Revuelta and M. Elices
}

\begin{abstract}
The lack of reliable mechanical data on coronary arteries and, more specifically, on their wall strength hampers the application of numerical models and simulations to vascular problems, and precludes physicians from knowing in advance the response of coronary arteries to the different interventions. Studies of the mechanical properties of coronary arteries have been carried out almost exclusively on animals. Only a few studies have tried to characterize the in vivo behavior of human coronaries through tests under physiological conditions. In this work, the mechanical properties of human coronary arteries have been characterized. Whole samples from human right (RC) and left anterior descending (LAD) coronary arteries aged between 23 and 83 years have been studied by means of in-vitro tensile testing up to failure.
\end{abstract}

\section{INTRODUCTION}

$\mathrm{T}_{\mathrm{p} m \mathrm{~s}}$ HE prevalence of coronary heart diseases in the world population has led to much research on this field. Coronary occlusions and ischemic heart diseases cause more than 7 millions of deaths every year.

Up to now, coronary reparation techniques, like by-pass surgery or angioplasty with stent implantation, do not take into account the mechanical properties of the coronary artery undergoing surgery. The lack of reliable mechanical data on coronary arteries and, more specifically, on their breaking conditions hampers the application of numerical models and simulations to real situations, and precludes physicians from knowing in advance the response of coronary arteries to the intervention.

Studies of the mechanical properties of coronary arteries have been carried out almost exclusively on animals [1]-[9]. Only a few studies [10]-[18] have tried to characterize the in vivo behavior of human coronaries through in vivo and in vitro tests under physiological conditions. Maybe the most complete in-vitro work has been carried out by Ozolanta et al. [12]; they studied in 205 patients the evolution with age of distensibility and modulus of elasticity of coronary arteries in the $0-240 \mathrm{mmHg}$ pressure range. Similar research was performed by Williams et al. [10] (in-vivo) and Tajaddini et al. [11] (in-vitro), which showed -in agreement with Ozolanta et al. [12]- that distensibility decreases and the modulus of elasticity increases with age.

Manuscript received April 23, 2010. This work was supported in part by the Comunidad de Madrid under MADR.IB-CM/S-SAL/0312/2006 program and by the Fundación Agustín de Betancourt in Spain.

E. Claes, J. M. Atienza, G. V. Guinea, F. J. Rojo and M. Elices are with the Materials Science Department of the Technical University of Madrid, 28040, Madrid, Spain (corresponding author to provide phone: 34913365239; fax: 34-913366680; e-mail: jmatienza@ mater.upm.es).

J. M. Bernal and J. M. Revuelta are with Servicio de Cirugía Cardiovascular, Hospital Marques de Valdecilla, Santander, Spain.
To the authors' knowledge, most of the mechanical studies on human coronary arteries in the literature have been limited to compliance and stiffness measurements not beyond twice or three times physiological pressure. Only Holzapfel et al [15] performed tensile tests up to failure of isolated intima, media and adventitia layers from 13 old human coronary arteries $(71.5 \pm 7.3 \mathrm{yr}$ old $)$, not reporting tests on whole specimens.

In this work, the mechanical properties of human coronary arteries have been characterized via tensile tests. To assess the mechanical resistance of coronary wall, whole samples from human right (RC) and left anterior descending (LAD) coronary arteries aged between 23 and 83 years have been studied by means of in-vitro tensile testing up to failure.

\section{MATERIALS}

Healthy RC and LAD coronary arteries were obtained from patients who died from non cardiovascular-related causes at the Hospital Marqués de Valdecilla (Santander, Spain). Coronary wall samples were obtained from 5 braindead donors aged between 23 and 83. In all cases, the tissues were obtained according to a protocol approved by the Hospital Ethics Committee, which included the informed consent of the next of kin. Despite their origin, histological analyses were performed on samples from all segments to corroborate the absence of vascular pathologies.

All the coronary samples were obtained with the maximum possible length $(>50 \mathrm{~mm})$ and preserved in saline solution at $4^{\circ} \mathrm{C}$ until testing within 24 hours from excision. The in vivo length of each vessel was marked before removal. In vivo longitudinal stretch was deduced from the ratio between in vivo and ex vivo length.

Donor information and vessel characteristics are summarized in Table I.

Table I. Donor information and vessel characteristics

\begin{tabular}{cccc}
\hline \hline Sex & Age $($ yr $)$ & Artery & Outer Diameter $(\mathbf{m m})$ \\
\hline \multirow{2}{*}{ Male } & 23 & $R C A$ & 3.84 \\
\cline { 3 - 4 } & & $L A D$ & 3.46 \\
Female & 35 & $L A D$ & 3.42 \\
\hline Male & 60 & $R C A$ & 5.14 \\
\hline Female & 63 & $L A D$ & 5.98 \\
\cline { 3 - 4 } & & $R C A$ & 5.88 \\
\hline \hline
\end{tabular}




\section{EXPERIMENTAL METHODS}

\section{A. Experimental set-up}

Uniaxial tensile tests were performed on rectangular specimens ( $1 \mathrm{~mm}$ wide $\times 7 \mathrm{~mm}$ long) stamped out in circumferential direction by means of a metal die of appropriate dimensions. Specimen thickness was measured on each sample before testing with a thickness gage with $10 \mu \mathrm{m}$ accuracy (Mitutoyo 7301). Thicknesses of coronary specimens ranged from 0.42 to $1.35 \mathrm{~mm}$.

Specimens were mechanically and chemically (glued with cyanoacrylate) fixed to the grips of an electromechanical tensile testing machine (Instron 5866) and immersed in saline solution at $37^{\circ} \mathrm{C}$. The temperature of the vessel was controlled to $0.5^{\circ} \mathrm{C}$ by a K-type thermocouple located in the chamber and close to the artery $(<4 \mathrm{~mm})$. The axial force was measured by means of a $10 \mathrm{~N}$ load cell (Instron 2519101, accuracy better than $5 \mathrm{mN}$ ) and the crosshead displacement was taken as a direct measurement of the elongation of the sample, as the compliance of the coronary specimen had been estimated to be 100 times greater than that of the equipment. The machine internal LVDT sensor (accuracy better than $10 \mu \mathrm{m}$ ) was used to measure the displacement of the crosshead. Tests were performed in the passive state, without electrical or chemical activation of the vascular wall. Loading rate was $0.03 \mathrm{~mm} / \mathrm{s}$ (approximately $36 \% /$ min strain rate). A detailed scheme of the testing procedure can be found elsewhere [19].

A minimum of three tensile tests for each artery were carried out. For the sake of clarity results are presented as patient's average.

\section{B. Evaluation of the mechanical parameters}

True stress $(\sigma)$ versus stretch $(\lambda)$ relationships for all the tests were derived from experimental load-displacement curves. The stretch $(\lambda)$ was obtained by dividing the current length (1) by the initial length (L) of the specimen, and the true stress $(\sigma)$ was calculated as the applied load $(\mathrm{P})$ divided by the current cross-sectional area (a). The hypothesis of tissue incompressibility was used to obtain the relationship between the current and the initial cross-sectional area (A), $\mathrm{a}=\mathrm{A} / \lambda$.

When tensile tested, arterial wall specimens typically display the characteristic J-shape curve shown in Fig. 1. For small values of applied stress, the aortic tissue exhibits a compliant behavior that turns into a much stiffer response when stresses exceed the elbow of the curve. To simplify the analysis and allow comparison among different specimens, the stress-stretch curve was condensed into three parameters that summarized the main mechanical response of the arterial wall: The stretch and stress at the breaking point $\left(\sigma_{\mathrm{R}}\right.$, $\left.\lambda_{R}\right)$, and the stress at the transition point, or "elbow", between the compliant and the stiff regions $\left(\sigma_{\mathrm{e}}\right)$.

The breaking point of the sample $\left(\sigma_{R}, \lambda_{R}\right)$ was defined as the first point where one of its layers fails, easily identified in the tensile curve by a sudden drop of the load. The elbow stress $\left(\sigma_{\mathrm{e}}\right)$ was determined by the change in the first derivative of stress with respect to stretch, according to the procedure given in the Appendix.

\section{RESULTS AND DISCUSSION}

\section{A. Tensile stress at the elbow point}

Fig. 1 sketches the stress-stretch curve of an arterial wall. Roach and Burton [20] proved in a classic study that the initial compliant zone for small and medium strains is due to elastin, while the much stiffer portion of the curve beyond the "elbow" is produced by the extension of collagen fibers. Depending on the circumferential stress level set off by blood pressure, the vessel wall will work in the compliant elastin-dominated part before the elbow or in the stiff collagen-dominated zone beyond it. Consequently, it is expected that variations in the mechanical behavior of the arterial wall produced by age or pathologies could lead to a change in the position of the elbow region and have a direct effect on arterial compliance throughout the cardiac cycle.

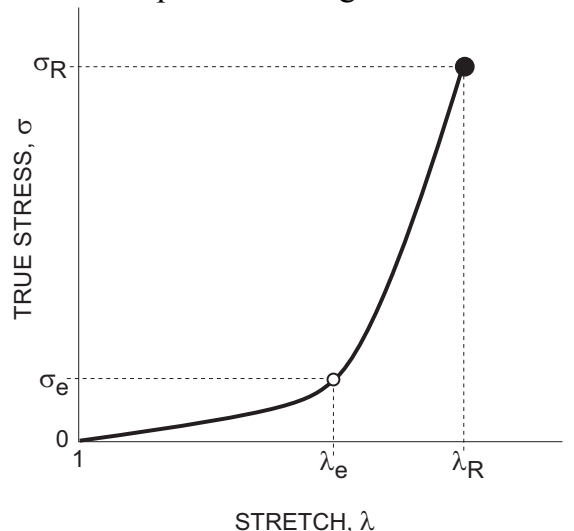

Fig. 1. Stress-stretch curve of a coronary artery and mechanical parameters

Fig. 2 plots the variation of elbow stresses as a function of age. Elbow stresses were computed from stress-stretch curves, as explained in the Appendix. Elbow stresses decrease monotonically with age. Also, Fig. 2 shows the mean "working" stress level computed under the assumption of normotensive pressure levels $(100 \mathrm{mmHg})$. Use of Laplace formula $\sigma=\mathrm{pD} / 2 \mathrm{t}$, where (p) is the blood pressure, (D) the aortic diameter and $(\mathrm{t})$ the wall thickness, taking into account the values of Table I, leads to in vivo mean circumferential stresses for patient.

Simple observation of data in Fig. 2 demonstrates that for the younger specimen the working zone of the coronary wall is well under the elbow stress. For the rest, the elbow stress is close to the working stress of the artery. These observations are coherent with published in vivo studies of aortic distensibility, which show a fall in arterial compliance with age [12]. Data on the wall distensibility could be used in the selection of the bypass graft in order to reduce the mechanical mismatch and therefore the stresses at the anastomosis. 


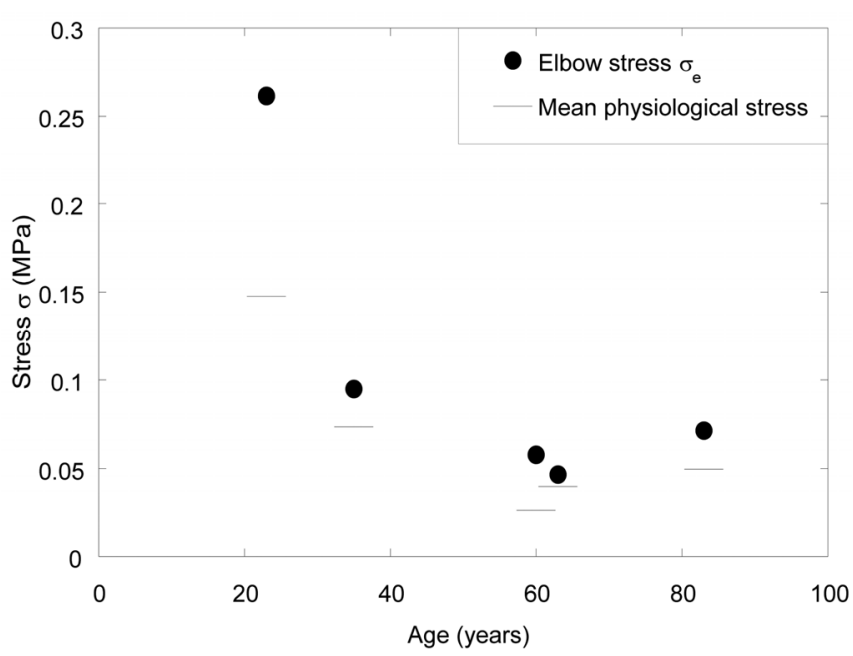

Fig. 2. Elbow stress of coronary arteries as a function of age and mean "working" stress at physiological conditions $(100 \mathrm{mmHg})$

\section{B. Coronary wall strength}

Fig. 3 displays the circumferential tensile strength $\left(\sigma_{R}\right)$ of coronary wall specimens as a function of age. To the authors' knowledge, this data on the mechanical strength of human coronary arteries are the first results on the whole arterial wall. The only work referring wall strength in coronaries is the study of Holzapfel et al [15], in which tensile tests were performed on separated intima, media and adventitia layers of coronaries arteries from 13 patients aged $72 \pm 7$ years. Interestingly, the results of the present work for the elder patients compare well with the breaking conditions of the media layer presented in that study; the circumferential tensile strength is $0.39 \pm 0.07 \mathrm{MPa}$ vs $0.45 \pm 0.19 \mathrm{MPa}$ from the referred study. Difficulties related to the obtaining of coronary vessels, which require a specialized dissection procedure due to their integration in the heart wall, and to their mechanical testing -due to their small dimensions- could be at the root of this lack of experimental data.

In comparison with other arteries, the values of circumferential strength of coronary arteries reported here are significantly lower than published results in ascending aorta, which share the elastic character with coronary arteries due to their proximity. Vorp et al. [21] found a circumferential strength of $1.80 \pm 0.24 \mathrm{MPa}$ for ascending arteries from $51 \pm 6$ years old donors and Iliopoulos et al. [22] reported $1.63 \pm 4 \mathrm{MPa}$ for a group aged $66 \pm 3$ years. These values are much higher than $0.39 \pm 0.07 \mathrm{MPa}$, found for coronary arteries of similar age.

Regarding the effect of age, the circumferential tensile strength of coronary arterial wall follows a decreasing trend, with a more or less sudden drop around the age of 30-40 years old. This trend correlates well with published results of mechanical properties of other arteries, like ascending [23] or descending aorta [24].

Up to now, decision on the level of balloon inflation pressure and the balloon size in an angioplasty does not take into account the mechanical properties of the coronary artery undergoing intervention. However, balloon inflation at high pressures can cause extensive arterial wall damage, with dissection and the creation of an occlusive intimal flap. The results of this work have helped to obtain the maximum admissible levels of pressure and dilatation imposed to coronary arteries during transluminal angioplasty [25], taking into consideration the actual arterial strength. As the balloon imposed a certain strain to what surrounds it, both arterial wall and atheroma plaque can be stretched depending on their relative stiffness. Therefore, knowledge on the mechanical properties of the arterial wall, combined with numerical simulations of the angioplasties could help cardiologists when deciding how far balloon can be inflated without damaging the artery.

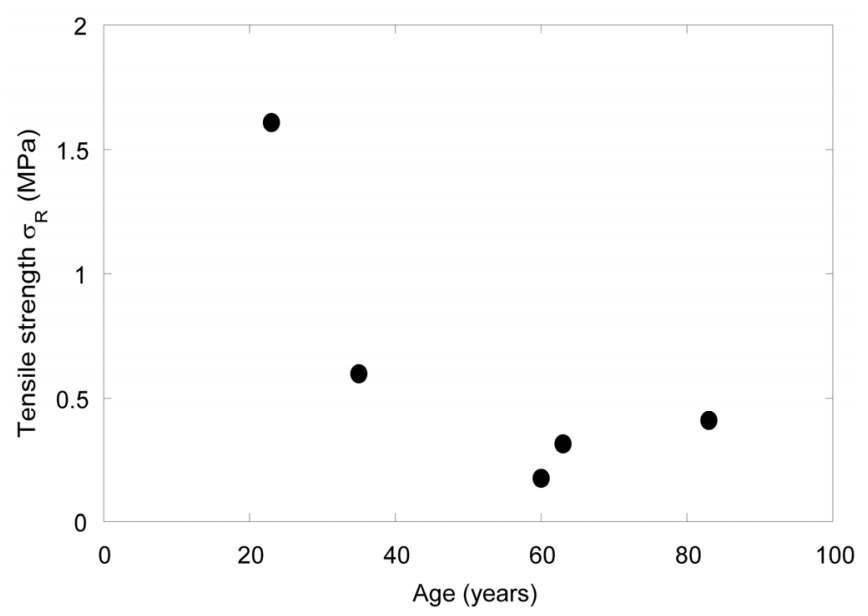

Fig. 3. Circumferential tensile strength of coronary wall specimens as a function of age

\section{CONCLUSION}

The mechanical properties of coronary arteries of 5 human donors have been studied by means of in-vitro tensile testing up to failure. Although the number of patients is scarce and a greater number of arteries of different ages must be studied to obtain better conclusions, the results could be of significant use because of the lack of data in literature. They can be useful in the application of numerical models and simulations predicting the response of coronary arteries to different surgery techniques. Data on the distensibility of coronary walls can serve to optimize the election of vascular grafts. Data on the tensile strength of coronary walls can help to prevent extensive arterial wall damage when balloon inflation reaches high pressures.

\section{APPENDIX: DETERMINATION OF THE ELBOW POINT OF THE STRESS-STRETCH CURVE.}

This appendix explains the method followed to obtain the coordinates of the transition point of the stress-stretch curve (Fig. A1). The procedure is based on the first derivative of the stress-stretch curve, d $\sigma / \mathrm{d} \lambda$ (Fig. A2), which was obtained numerically for each specimen by means of the 
software "Kaleidagraph(C)" On the $\mathrm{d} \sigma / \mathrm{d} \lambda$ curve, the transition zone is readily identified as the nearly linear central region before the upper plateau, as shown in Fig. A2. The elbow point is defined as the mid-point of the transition zone (Fig. A2). The stretch at the elbow $\left(\sigma_{\mathrm{e}}\right)$ is then determined on the $\mathrm{d} \sigma / \mathrm{d} \lambda$ curve and the corresponding stress $\left(\sigma_{\mathrm{e}}\right)$ is obtained directly from the stress-stretch curve.

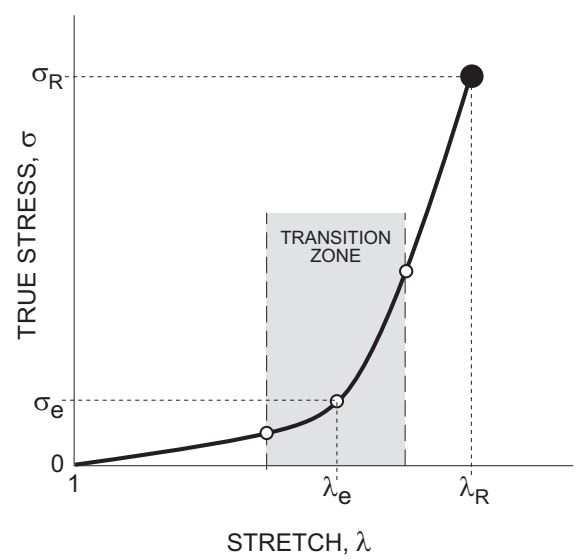

Fig. A1. Stress-stretch curve of the arterial wall

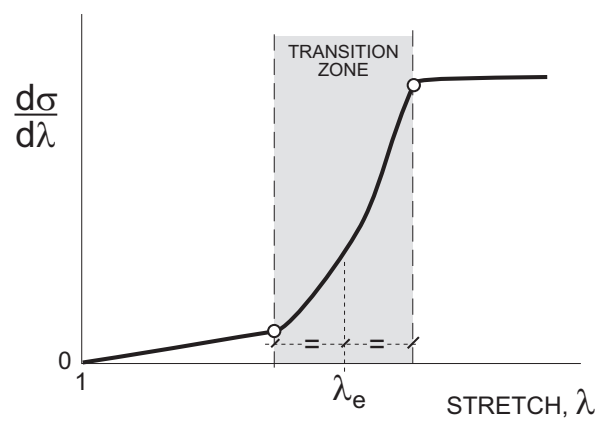

Fig. A2. First derivative of the stress-stretch curve

\section{REFERENCES}

[1] M. Szekeres, G. L. Nadasy, L. Dezsi, M. Orosz, A. Tokes, and E. Monos, "Segmental differences in geometric, elastic and contractile characteristics of small intramural coronary arteries of the rat," Journal of Vascular Research, vol. 35, pp. 332-344, 1998.

[2] C. Lally, A. J. Reid, and P. J. Prendergast, "Elastic behavior of porcine coronary artery tissue under uniaxial and equibiaxial tension," Annals of Biomedical Engineering, vol. 32, pp. 1355-1364, 2004.

[3] C. Wang, W. Zhang, and G. S. Kassab, "The validation of a generalized Hooke's law for coronary arteries," American Journal of Physiology-Heart and Circulatory Physiology, vol. 294, pp. H66-H73, 2008.

[4] S. J. Bund, A. A. Oldham, and A. M. Heagerty, "Mechanical properties of porcine small coronary arteries in one-kidney, one-clip hypertension," Journal of Vascular Research, vol. 33, pp. 175-180, 1996.

[5] B. S. Gow, D. Schonfel, and D. J. Patel, "Dynamic Elastic Properties of Canine Left Circumflex Coronary-Artery," Journal of Biomechanics, vol. 7, pp. 389-395, 1974.

[6] D.J. Patel and J. S. Janicki, "Static Elastic Properties of Left Coronary Circumflex Artery and Common Carotid Artery in Dogs," Circulation Research, vol. 27, pp. 149-158, 1970.

[7] A. I. Veress, D. G. Vince, P. M. Anderson, J. F. Cornhill, E. E. Herderick, B. D. Kuban, N. L. Greenberg and J. D. Thomas, "Vascular mechanics of the coronary artery," Zeitschrift Fur Kardiologie, vol. 89, pp.92-100, 2000.

[8] J. G. De Mey and D. L. Brutsaert, "Mechanical properties of resting and active isolated coronary arteries," Circ Res, vol. 55, pp. 1-9, 1984.
[9] C. J. van Andel, P. V. Pistecky, and C. Borst, "Mechanical properties of coronary arteries and internal mammary arteries beyond physiological deformations," in Proc. 23rd Annual International Conference of the Ieee Engineering in Medicine and Biology Society, vol. 1-4, no. 23, pp. 113-115, 2001.

[10] M. J. A. Williams, R. A. H. Stewart, C. J. S. Low, and G. T. Wilkins, "Assessment of the mechanical properties of coronary arteries using intravascular ultrasound: an in vivo study," International Journal of Cardiac Imaging, vol. 15, pp. 287-294, 1999.

[11] A. Tajaddini, D. L. Kilpatrick, P. Schoenhagen, E. M. Tuzcu, M. Lieber, and D. G. Vince, "Impact of age and hyperglycemia on the mechanical behavior of intact human coronary arteries: an ex vivo intravascular ultrasound study," American Journal of PhysiologyHeart and Circulatory Physiology, vol. 288, pp. H250-H255, 2005.

[12] I. Ozolanta, G. Tetere, B. Purinya, and V. Kasyanov, "Changes in the mechanical properties, biochemical contents and wall structure of the human coronary arteries with age and sex," Medical Engineering \& Physics, vol. 20, pp. 523-533, 1998.

[13] V. Kasjanovs, I. Ozolanta, and B. Purina, "Features of biomechanical properties of human coronary arteries," Mechanics of Composite Materials, vol. 35, pp. 155-168, 1999.

[14] C. J. van Andel, P. V. Pistecky, and C. Borst, "Mechanical properties of porcine and human arteries: Implications for coronary anastomotic connectors," Annals of Thoracic Surgery, vol. 76, pp. 58-64, 2003.

[15] G. A. Holzapfel, G. Sommer, C. T. Gasser, and P. Regitnig, "Determination of layer-specific mechanical properties of human coronary arteries with nonatherosclerotic intimal thickening and related constitutive modeling," American Journal of Physiology-Heart and Circulatory Physiology, vol. 289, pp. H2048-H2058, 2005.

[16] B. S. Gow and C. D. Hadfield, "Elasticity of Canine and Human Coronary-Arteries with Reference to Postmortem Changes," Circulation Research, vol. 45, pp. 588-594, 1979.

[17] O. S. Opgaard and L. Edvinsson, "Mechanical properties and effects of sympathetic co-transmitters on human coronary arteries and veins," Basic Research in Cardiology, vol. 92, pp. 168-180, 1997.

[18] D. V. Carmines, J. H. McElhaney, and R. Stack, "A Piece-Wise Nonlinear Elastic Stress Expression of Human and Pig CoronaryArteries Tested Invitro," Journal of Biomechanics, vol. 24, pp. 899906, 1991.

[19] G. Guinea, J. Atienza, P. Fantidis, F. Rojo, A. Ortega, M. Torres, P. Gonzalez, M. Elices, K. Hayashi, and M. Elices, "Increases of Corporal Temperature as a Risk Factor of Atherosclerotic Plaque Instability," Annals of Biomedical Engineering, vol. 36, pp. 66-76, 2008.

[20] M. R. Roach and A. C. Burton, "The Reason for the Shape of the Distensibility Curves of Arteries," Canadian Journal of Biochemistry and Physiology vol. 35, no. 8, pp. 681-690, 1957.

[21] D. A. Vorp, B. J. Schiro, M. P. Ehrlich, T. S. Juvonen, M. A. Ergin, and B. P. Griffith, "Effect of aneurysm on the tensile strength and biomechanical behavior of the ascending thoracic aorta," Annals of Thoracic Surgery, vol. 75, pp. 1210-1214, 2003.

[22] D. C. Iliopoulos, E. P. Kritharis, A. T. Giagini, S. A. Papadodima, and D. P. Sokolis, "Ascending thoracic aortic aneurysms are associated with compositional remodeling and vessel stiffening but not weakening in age-matched subjects," Journal of Thoracic and Cardiovascular Surgery vol. 137, pp. 101-109, 2009.

[23] R. J. Okamoto, J. E. Wagenseil, W. R. DeLong, S. J. Peterson, N. T. Kouchoukos, and T. M. Sundt, "Mechanical properties of dilated human ascending aorta," Annals of Biomedical Engineering vol. 30, pp. 624-635, 2002.

[24] J. P. Vande Geest, M. S. Sacks, and D. A. Vorp, "Age dependency of the biaxial biornechanical Behavior of humian abdominal aorta," Journal of Biomechanical Engineering-Transactions of the Asme vol. 126, pp. 815-822, 2004.

[25] E. Claes, G. V. Guinea, F. J. Rojo, J. M. Atienza, J. M. Bernal, J. M. Revuelta, and M. Elices, "Assessment of maximum pressure and lumen diameter to prevent coronary dissection during balloon angioplasty," International Journal of Cardiology, under revision. 